Didacticiel du style de citation APA 



\title{
Didacticiel du style de citation APA
}

\author{
ze édition
}

SARAH ADAMS AND DEBBIE FEISST

NORQUEST COLLEGE LIBRARY AND

ALISSA DROOG

\author{
UNIVERSITY OF ALBERTA LIBRARY \\ EDMONTON
}




\section{() (1) () (2)}

Didacticiel du style de citation APA by University of Alberta Library is licensed under a Creative Commons Attribution-NonCommercial-ShareAlike 4.0 International License, except where otherwise noted.

Le didacticiel du style de citation APA de la Bibliothèque de l'université de l'Alberta est une traduction et adaptation de APA Citation Style Tutorial qui est lui-même une adaptation de Introduction to APA Library Tutorials de NorQuest College Library licensed under a Creative Commons Attribution-Pas d'utilisation commerciale-Partage dans les mêmes conditions 4.0 International License.

This book was produced with Pressbooks (https://pressbooks.com) and rendered with Prince. 


\section{Contents}

Introduction 1

Part I. Pourquoi citer?

Pourquoi citer? $\quad 5$

Un aperçu du style de citation APA (7e édition) 11

Part II. Les sortes de sources d'information

Les sortes de sources d'information $\quad 15$

Les sortes de sources : Les repères d'articles de 17 revues

Les sortes de sources : Les repères de publications $\quad 20$ professionnelles

Les sortes de sources : Repères de page Web 22

Les sortes de sources : Repères de livre imprimé 23 et électronique

Résumé des sortes de sources $\quad 25$

Part III. Les références bibliographiques

Deux sortes de citations $\quad 29$

Qu'est-ce qu'une liste de références 31

bibliographiques?

La préparation des références bibliographiques 
Activité pour la préparation des références

bibliographiques

Part IV. Citations dans le texte

Qu'est-ce qu'une citation ou un renvoi dans le texte?

Activité pour les citations dans le texte

Solutions à l'activité pour les citation dans le texte

Part V. Comment tout fonctionne ensemble

Comment tout fonctionne ensemble 59

Activité de couplage des références

bibliographiques et des citations dans le texte

APA et aide à la rédaction

65

Conclusion

68

Références bibliographiques et attribution 


\section{Introduction}

Bienvenue au tutoriel sur le style de citation APA pour les cours en éducation créé par la bibliothèque de l'Université de l'Alberta. Dans ce didacticiel, vous vous familiariserez avec les règles de citation de la 7e édition de la APA.

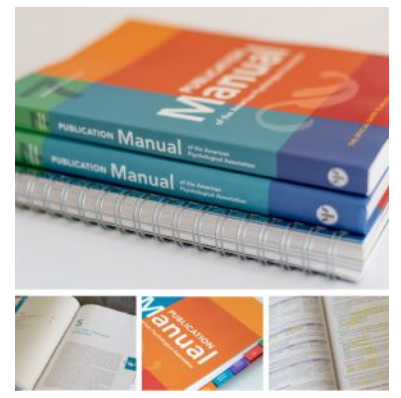

A la suite de ce tutoriel, vous pourrez :

1. Identifier pourquoi il est important de citer,

2. Reconnaître les indices qui indiquent la sorte de source citée,

3. Créer et agencer les éléments d'une liste de références et de citations dans le texte selon le style APA,

4. Consulter des ressources d'appui qui aident à 
utiliser le style de citation APA.

Image de: Publication Manual of the American Psychological Association, 7th edition. 
PART I

\section{POURQUOI CITER?}

Pourquoi citer? | 3 



\section{Pourquoi citer?}

A la suite de ce chapitre, vous pourrez :

- Identifier les raisons pour lesquelles citer est important.

Il est important de comprendre pourquoi les citations sont fondamentales à tout devoir de recherche, en plus d'être une exigence. Une citation est une référence à une source qui contient des informations clés essentielles à sa localisation.

Voici 3 raisons clées pour lesquelles il est important de citer.

1re Raison : La communication savante 


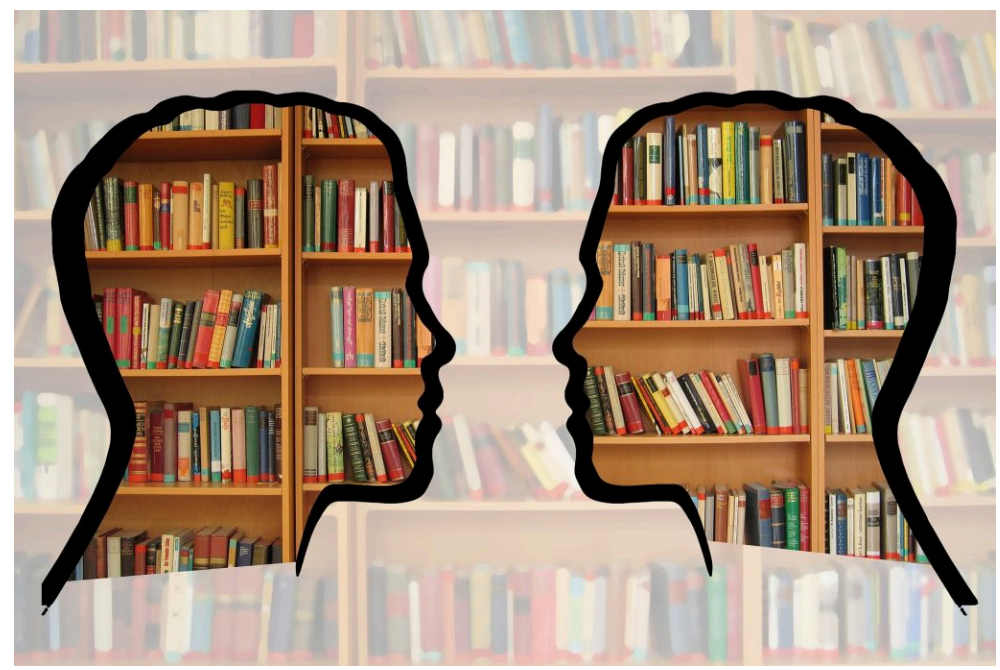

En lisant, en analysant et en incluant des sources érudites dans vos devoirs, vous contribuez et participez à la communication savante!

Vous développez votre compréhension d'un domaine d'études en apprenant de ses experts en la matière. En tant qu'enseignants, vous appliquez la recherche pédagogique pour améliorer vos pratiques d'enseignement et vous vous conformer à la norme de qualité d'enseignement 2d d'Alberta Education.

2e Raison : Reconnaître les contributions des autres et faire preuve de déontologie 


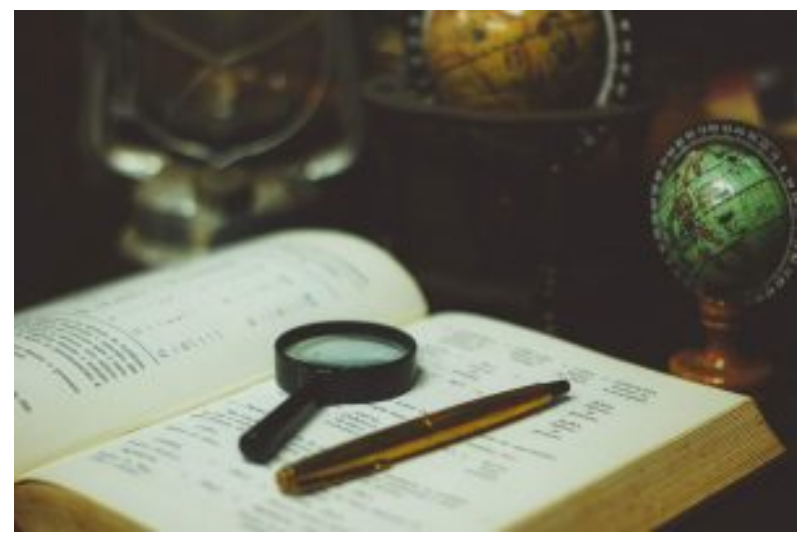

Citer dans une dissertation est essentiel pour démontrer du professionnalisme et donner des preuves.

Vous utiliserez principalement des sources universitaires et professionnelles comme preuves pour soutenir vos recherches et reconnaître les contributions de ces mêmes sources. Les citations permettent aux autres (et à vous!) de retrouver les sources utilisées dans votre dissertation pour en savoir davantage sur elles.

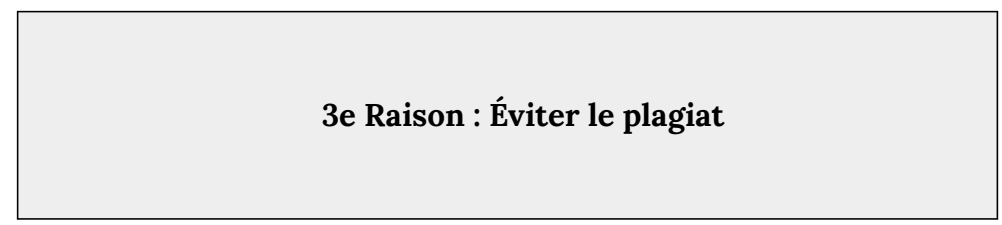




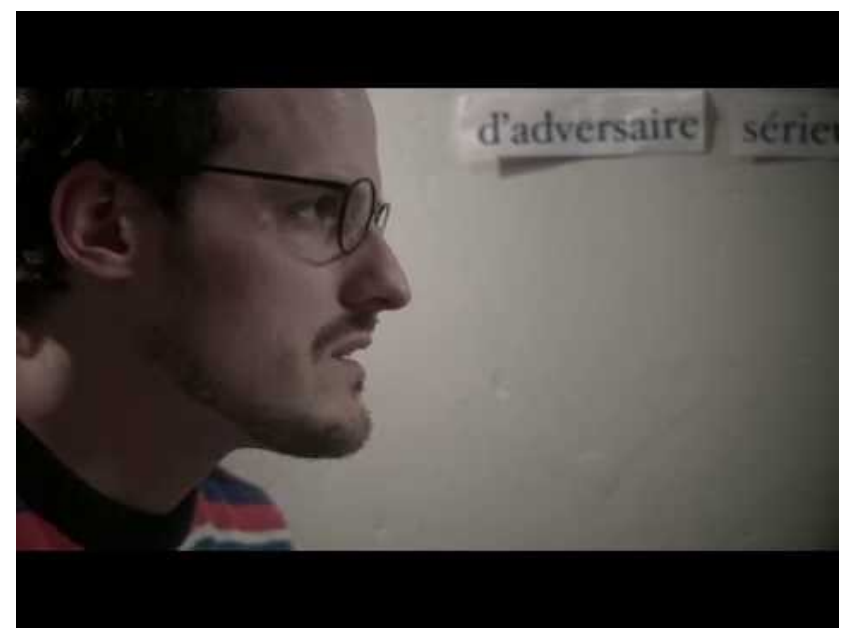

A YouTube element has been excluded from this version of the text. You can view it online here:

https://openeducationalberta.ca/tutoriel-apa/?p=22 


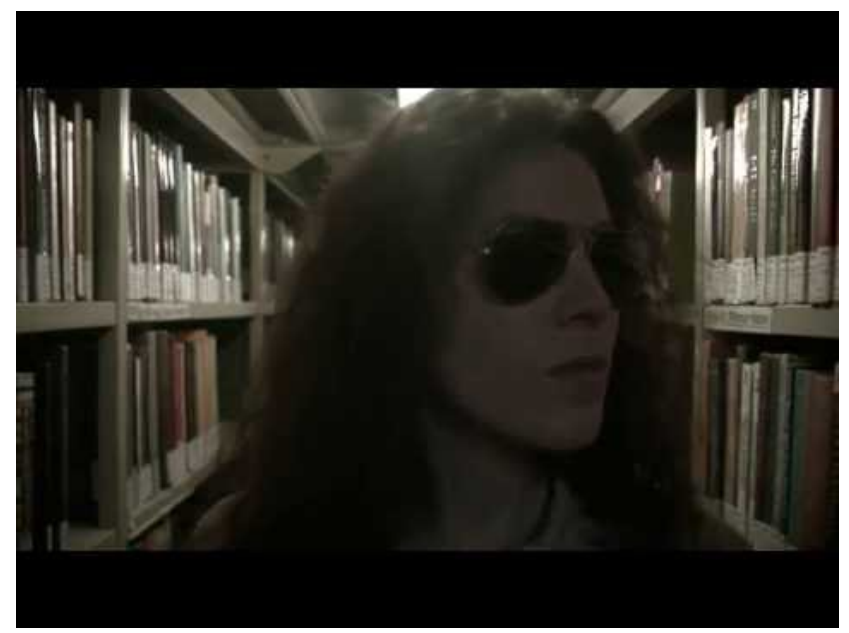

A YouTube element has been excluded from this version of the text. You can view it online here:

https://openeducationalberta.ca/tutoriel-apa/?p=22

Regardez ces deux courtes vidéos pour en savoir plus sur le plagiat et comment l'éviter.

La question suivante permet de réfléchir à l'origine des sources utilisées : Est-ce ma propre idée ou ai-je lu ou entendu parler de cela dans l'une de mes sources? Si les informations viennent d'ailleurs, citez-les.

En résumé

Citer permet de : 
1. Participer à la communication savante,

2. Reconnaître les contributions d'autrui,

3. Éviter le plagiat.

Attribution:

"[What exactly is citing summary]" de NorQuest College Library's Intro to APA tutorials selon la licence CC BY-NC-SA 4.0 International. 


\section{Un aperçu du style de citation APA (7e édition)}

Le style de citation APA est un ensemble normalisé de règles de formatage et de citation utilisées dans les sciences sociales et les programmes professionnels, tels que l'éducation. Il comprend des directives pour le formatage de votre article (formatage du document), la citation de sources dans le texte de votre article (citations dans le texte) et la citation de sources à la fin de votre article (citations de la liste de références).

La 7e édition des normes de l'APA comprend quelques changements clés, en particulier pour les sources en ligne.

Résumé des nouveautés de la 7e édition du style de citation APA (compilées à partir du guide de l'Université de Montréal)

- Dans la liste de références :

- Le lieu de publication n'est plus inclus dans les références de livres et de chapitres de livres.

- Toujours indiquer l'adresse Internet permanente de la ressource électronique citée en forme de DOI (e.g. https://doi.org/... plutôt que l'URL) lorsque celui-là est disponible. Le préfixe doi: qui précédait l'adresse Internet n'est plus utilisé.

- Le préfixe Repéré à qui était placé devant l'adresse Internet n'est plus utilisé.

- Les titres des pages Web individuelles, des publications sur les médias sociaux et des messages publiés sur les forums de discussion apparaissent maintenant en caractère italique.

- Mentionner tous les noms d'auteur dans la référence jusqu'à 20 auteurs. À partir de 21 auteurs, inscrire les 19 
premiers, suivi de points de suspension (...) puis du dernier auteur cité.

- La date de dernière consultation n'est plus mentionnée dans la référence, sauf dans le cas des publications modifiées ou mises à jour très fréquemment, comme les médias sociaux (Facebook, Twitter, etc.).

- Lorsque l'auteur et l'éditeur sont la même entité, celle-ci n'est dorénavant mentionnée qu'en position d'auteur (on omet l'information en position d'éditeur).

- Dans les citations dans le texte :

- Pour les références ayant un ou deux auteurs, inclure tous les noms dans chaque citation. Pour les références ayant trois auteurs ou plus, inclure le nom du premier auteur, suivi de « et al. », et ce, dès la première citation.

Voir aussi la vidéo (en anglais) des nouveautés dans le style de citation APA.

Références aux médias utilisés :

Image de : Publication Manual of the American Psychological Association, 7th edition.

Scribbr. (2019, November 26). APA manual 7th edition: 17 most notable changes | Scribbr [Video]. YouTube. https://www.youtube.com/watch?v=zeSIXD6y3WQ

Université de Montréal. (2020). Nouveautés 7e édition Citer selon les normes de l'APA $7 e$ édition. https://bib.umontreal.ca/citer/styles-bibliographiques/ apa?tab=3282\#

12 | Un aperçu du style de citation APA (7e édition) 
PART II

\section{LES SORTES DE SOURCES D'INFORMATION}





\section{Les sortes de sources d'information}

Résultat d'apprentissage

A la suite de ce chapitre, vous pourrez :

- Reconnaître les repères qui permettent d'identifier la sorte de source.

Pour identifier les bons éléments du style de citation APA dans une liste de références, il est nécessaire d'abord de savoir de quelle sorte de source il s'agit. L'examen des sources à partir d'indices visuels ou de description dans un catalogue de bibliothèque ou une base de données peut vous aider à déceler les éléments de style nécessaires.

Maintenant que tant de sources sont en ligne, il peut être un peu difficile de déterminer de quelle sorte de source il s'agit. Pour vous aider, nous allons passer en revue quelques exemples de sources et d'indices à identifier. 


\section{Sources populaires et savantes}

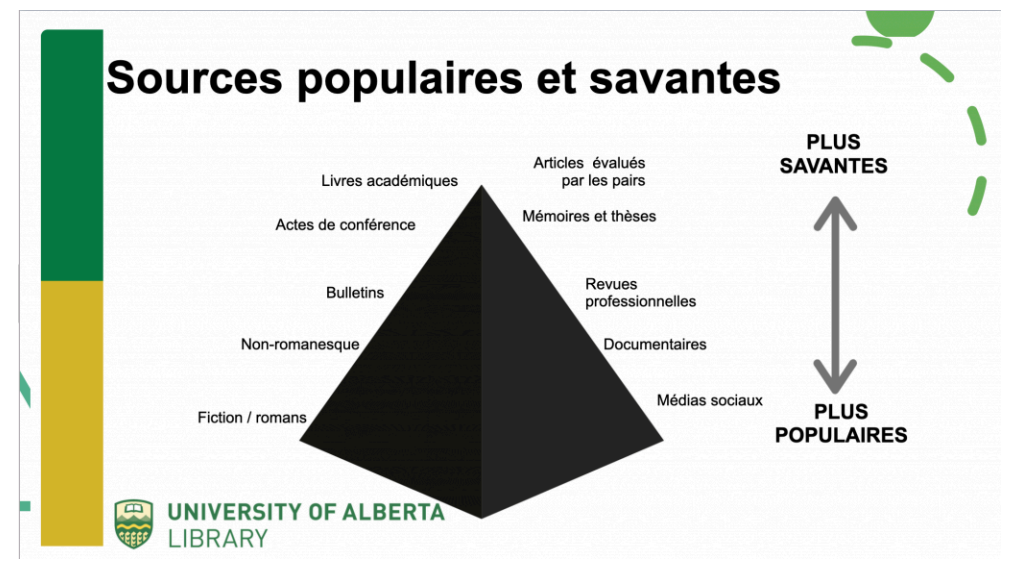

16 | Les sortes de sources d'information 


\section{Les sortes de sources : Les repères d'articles de revues}

Dans les exemples de sortes de sources suivants, examinez les différentes sources et cliquez sur le symbole $\mathbf{i}_{\text {pour en savoir plus }}$ sur chaque repère qui aide à identifier la sorte de source dont il s'agit.

Tout d'abord, nous examinerons un article de revue scientifique. 


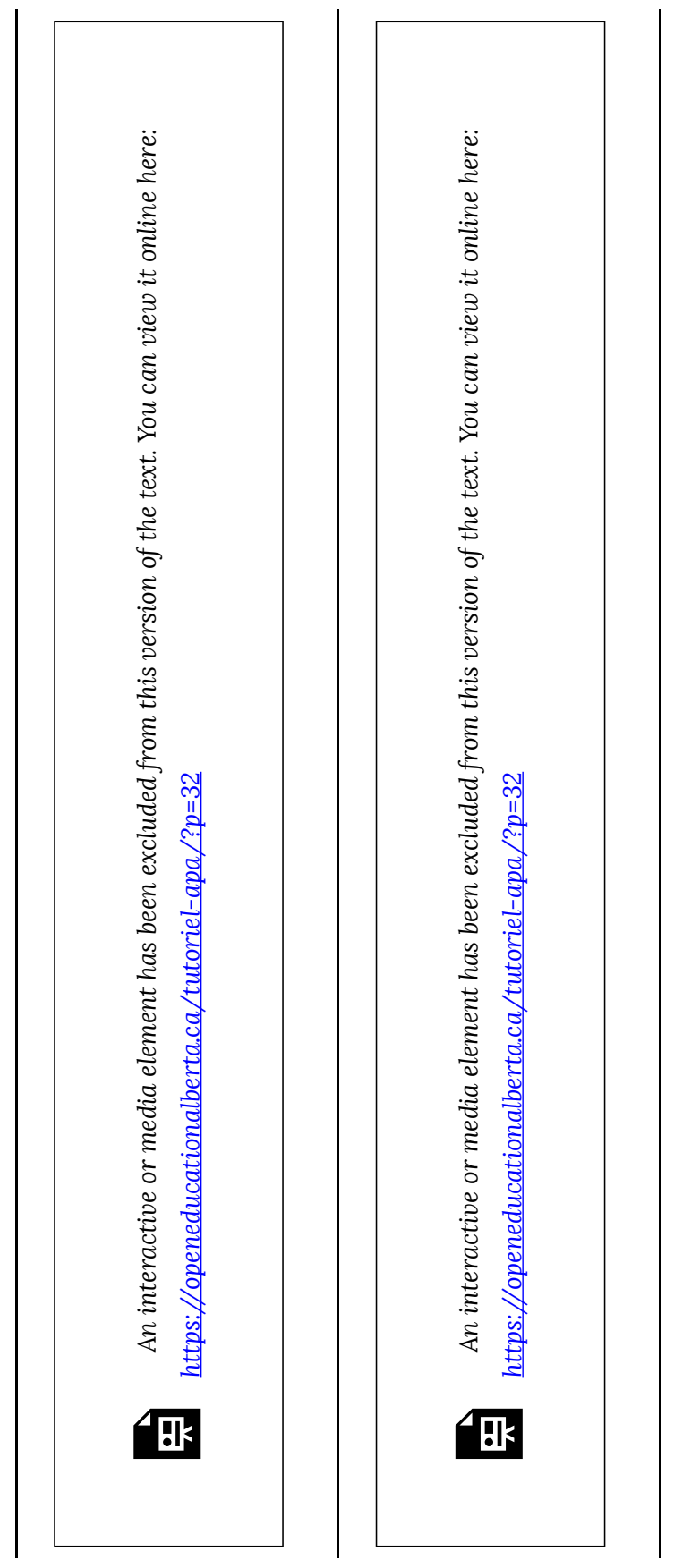




\section{Citation des ouvrages:}

Pellerin, M. (2017). L'usage des technologies numériques pour le développement de compétences multimodales en littératie au $21^{\mathrm{e}}$ siècle. Éducation et francophonie, 45(2), 85-106. https://doi-org.login.ezproxy.library.ualberta.ca/10.7202/ $\underline{1043530 \mathrm{ar}}$

Tardif, C., \& McMahon, F. (1989). Les francophones et les etudes postsecondaires (Francophones and Postsecondary Studies). Canadian Journal of Higher Education, 19(3), 19-28. 


\title{
Les sortes de sources : Les
}

\section{repères de publications professionnelles}

Examinez la source et cliquez sur le symbole $\mathbf{D}$ pour en savoir plus sur chaque repère qui aide à identifier une publication professionnelle.
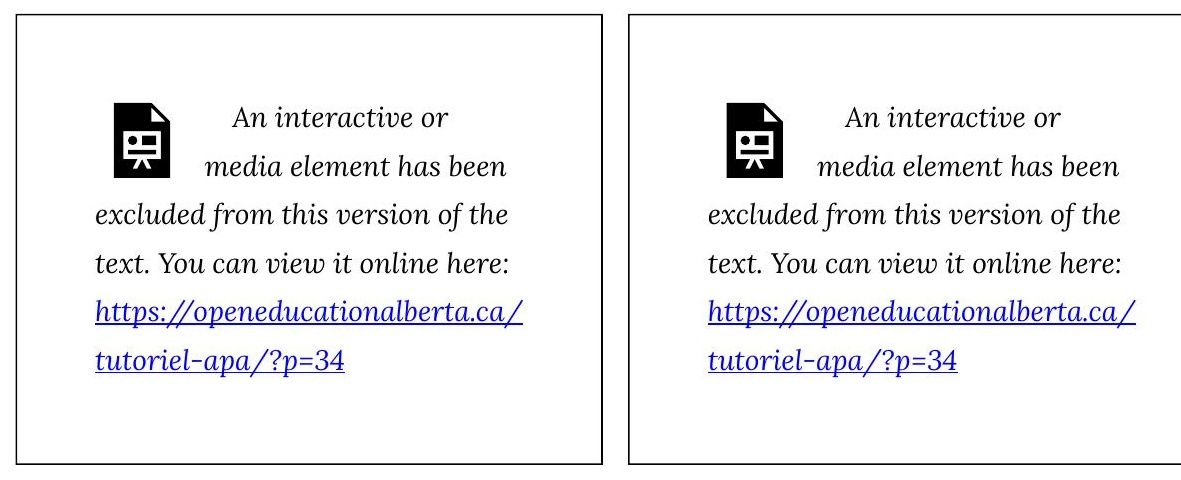

\begin{abstract}
An interactive or media element has been excluded

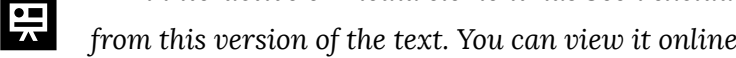

here:

https://openeducationalberta.ca/tutoriel-apa/?p=34

\section{Citation de l'ouvrage:}

Carpentier, G. (2020, juin). La bienveillance ne doit jamais être en 
confinement. Vivre le primaire, 5. https://aqep.org/wp-content/ uploads/2020/06/La-bienveillance-ne-doit-jamais-etre-enconfinement.pdf 


\section{Les sortes de sources : Repères de page Web}

Examinez la source et cliquez sur le symbole $\mathbf{1}$ pour en savoir plus sur chaque repère qui permet d'identifier une page Web.

\section{Citation de l'ouvrage:}

Canadian Parents for French. (2020). Accueil. https://cpf.ca/fr/ ressources/pour-parents/ 


\section{Les sortes de sources : Repères de livre imprimé et électronique}

Examinez la source et cliquez sur le symbole $\mathbf{B}$ pour en savoir un peu plus sur les repères qui permettent d'identifier un livre imprimé et électronique.

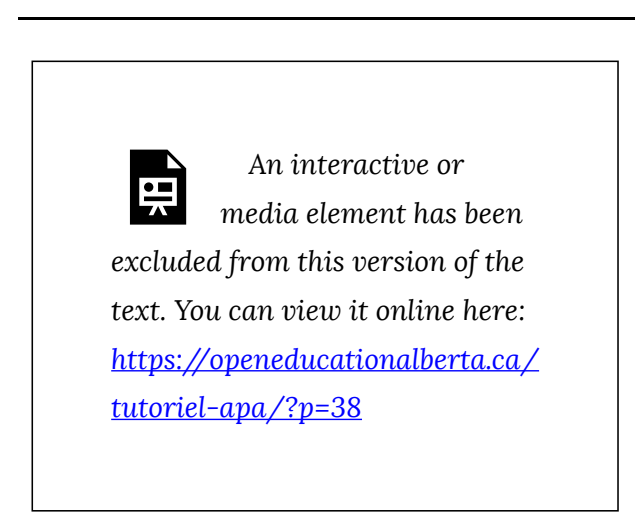


$\Delta$ An interactive or

둣 media element has been excluded from this version of the text. You can view it online here: https://openeducationalberta.ca/ tutoriel-apa $/ ? p=38$
An interactive or media element has been excluded from this version of the text. You can view it online here: https://openeducationalberta.ca/ tutoriel-apa/?p=38

\section{Citation de l'ouvrage:}

Gohier, C. (2007). Identités professionnelles d'acteurs de l'enseignement : regards croisés. Québec: Presses de l'Université du Québec. 


\section{Résumé des sortes de sources}

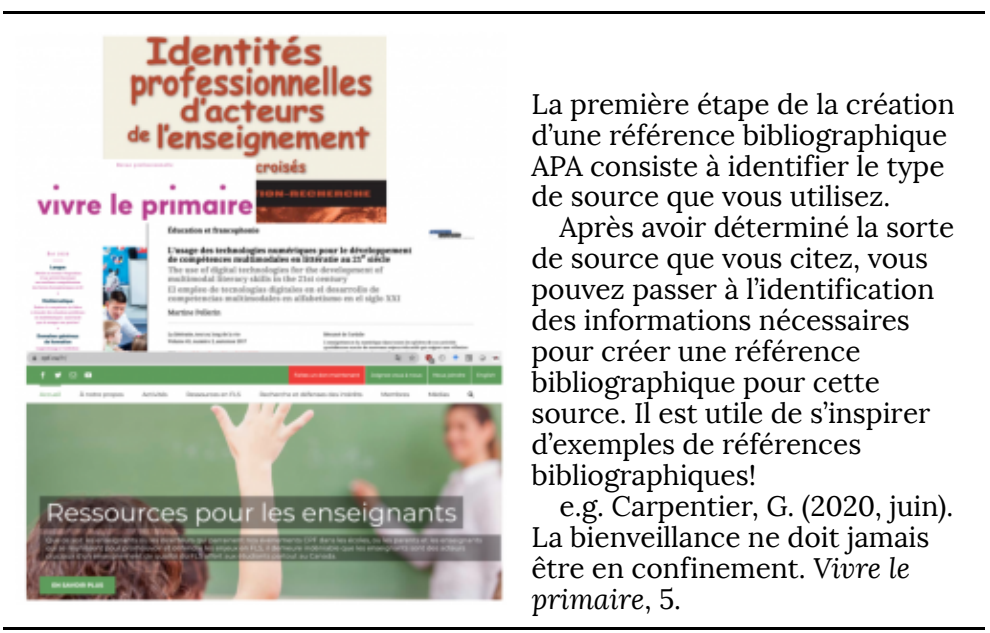





\section{PART III \\ LES RÉFÉRENCES BIBLIOGRAPHIQUES}





\section{Deux sortes de citations}

A la suite de ce chapitre, il vous sera possible de :

- Créer et de formater une liste de références bibliographiques dans le style APA.

Dans le style de citation APA, il y a deux parties à chaque citation : un renvoi ou une citation dans le texte et une liste de références bibliographiques correspondantes. 


\section{Les références}

bibliographiques sont des citations plus longues qui fournissent suffisamment d'informations pour décrire et retrouver votre source, physiquement ou en ligne.
American Psychiatric Association. (2015). DSM-5 : manuel diagnostique et statistique des troubles mentaux (traduit par J.-D. Guelfi et M.-A. Crocq; 5e éd.). Elsevier Masson.

Chouchena, O., Soulé, M. et Noël, J. (2004). Les grands-parents dans la dynamique normale ou pathologique des enfants. Dans S. Lebovici, R. Diatkine et M. Soulé (dir.), Nouveau traité de psychiatrie de l'enfant et de l'adolescent (2e éd., vol. 4, p. 2633-2660). Presses universitaires de France.

Leduc, L. (2019, 5 novembre). DPJ : les enfants risquent d'être traumatisés de nouveau par le système. La Presse.

https://www.lapresse.ca/actualites/201911/05/01-5248405-dpj-les-enfants-risquent-detre-traumat ses-de-nouveau-par-le-systeme.php

Préfontaine, I. et Lanovaz, M. J. (2017). Intervention psychosociale en autisme : examen de la portée de la littérature francophone. Revue de psychoéducation, 46(2), 301-312. https://doi-org.login.ezproxy.library.ualberta.ca/10.7202/1042252ar

\section{Un renvoi dans le texte}

est une forme abrégée d'une citation qui apparaît dans une liste de références et par laquelle une source est reconnue dans votre dissertation. La citation abrégée présente suffisamment

Une étude (Préfontaine et Lanovaz, 2017) a démontré que [...] OU

En 2017, une étude (Préfontaine et Lanovaz) a démontré que [...] ou

Préfontaine et Lanovaz (2017) ont démontré dans une étude que [...] d'informations pour en localiser sa forme plus étoffée dans la liste de références.

Nous discuterons de ces deux cas séparément d'abord, puis ensemble plus tard. Dans le prochain chapitre, nous discuterons des listes de références bibliographiques. 


\section{Qu'est-ce qu'une liste de références bibliographiques?}

Commençons par discuter de ce qu'est une liste de références bibliographiques. Il s'agit d'une liste alphabétique incluse à la fin de votre article qui répertorie toutes les citations aux sources utilisées pour soutenir votre recherche dans votre dissertation.

Chaque référence comprend des éléments clés qui répondent à $\mathbf{4}$

\section{Questions:}

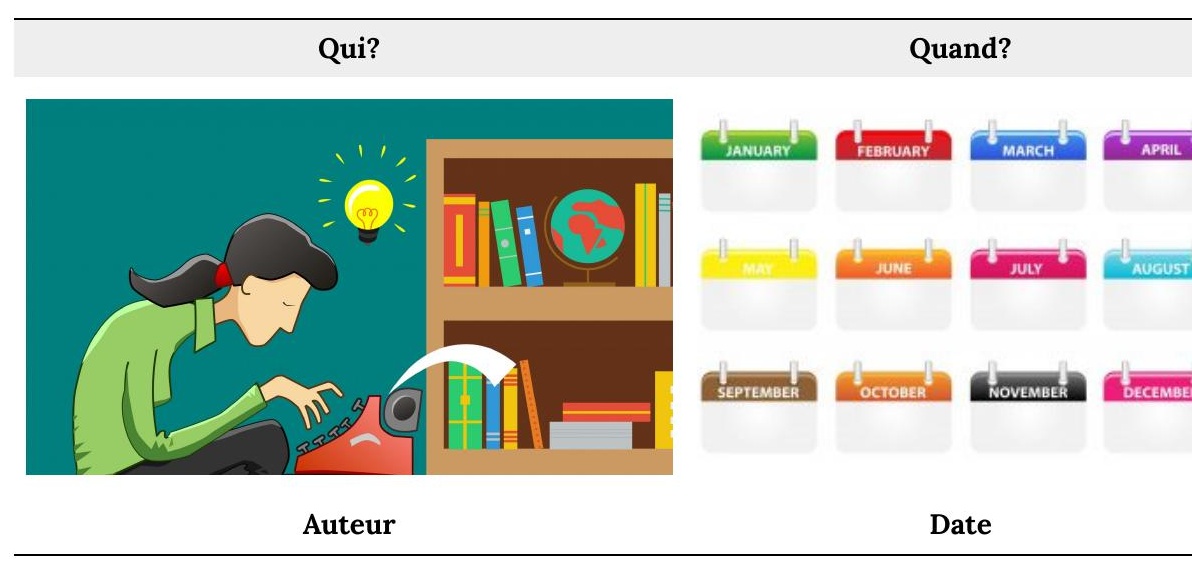

Les 4 questions permettent d'identifier les éléments clés nécessaires pour la construction d'une référence bibliographique.

On ne s'attend pas à ce que vous mémorisiez les consignes de l'APA. Utilisez plutôt les ressources disponibles (Aide mémoire APA, le guide de citation APA et bien sûr ce tutoriel) pour vous guider. Avec le temps, vous deviendrez plus à l'aise pour créer vous-même des citations et des références bibliographiques. 


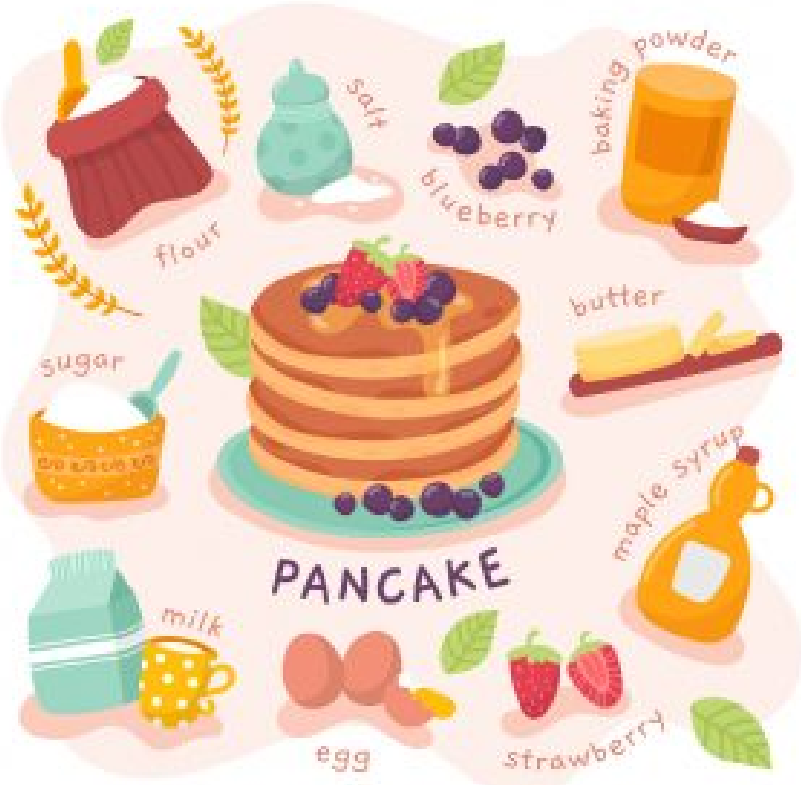

Utiliser les ressources APA pour aider à créer une référence bibliographique revient à utiliser une recette. Les ingrédients sont les informations clés qui décrivent une source en réponse aux 4 questions. S'il vous manque un ingrédient, omettez-le ou remplacez-le.

Suivre les instructions d'une recette est semblable à suivre un exemple prédéterminé de référence bibliographique. Si vous suivez les instructions et ajoutez les ingrédients au bon endroit, votre recette (c'est-à-dire, votre citation) réussira!

Attributions d'image:

"stack-of-food-books-2" by Cannelle is licensed under CC BY 4.0 International. “512px-Internet2" by Fabio Lanari is licensed under CC BY-SA 4.0 International.

32 | Qu'est-ce qu'une liste de références bibliographiques? 
"Pancake recipe with ingredients Free Vector" by pikisuperstar is licensed under CC BY 4.0 International. 


\section{La préparation des références bibliographiques}

Ensuite, nous utiliserons les 4 Questions (4Q) pour examiner chaque référence bibliographique aux ouvrages donnés comme exemple dans la section «Explorez les sortes de sources». Faites très attention à la ponctuation et à la police (italique, soulignement) utilisées pour chaque sorte de source.

L'aide-mémoire APA de la bibliothèque est une excellente ressource à avoir sous la main pour créer des références bibliographiques.

Dans l'image ci-dessus, servez-vous des flèches de la barre de défilement afin de passer les 4 images en revue.

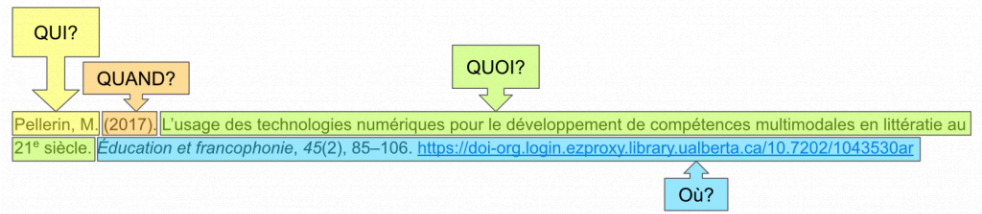

34 | La préparation des références bibliographiques 
L'exemple ci-dessus est une référence typique à un article de revue avec DOI (identificateur d'objet numérique).

- Auteur : est le nom de famille et l'initiale du prénom pour chaque auteur, séparés par une virgule et une esperluette avant le dernier auteur. (Dans cet exemple, il n'y a qu'un seul auteur.)

- Les noms des auteurs sont organisés dans l'ordre qu'ils apparaissent dans l'ouvrage.

- Date : est la date de publication (année) de l'article.

- Titre : tous les titres dans le style APA commencent avec une majuscule. De même, sont en majuscule le premier mot d'un sous-titre et tous les noms propres.

- Localisation de la source : se réfère au titre de la revue érudite (pour les périodiques en français, mettre une majuscule à la première lettre du premier mot du titre; pour les périodiques en anglais, mettre tous les mots en majuscule sauf les petits mots, en italique), volume et numéro, intervalle de pages, lien DOI.

- Larticle fait partie d'une source plus grande (la revue érudite), donc la source est en italique et non l'article.

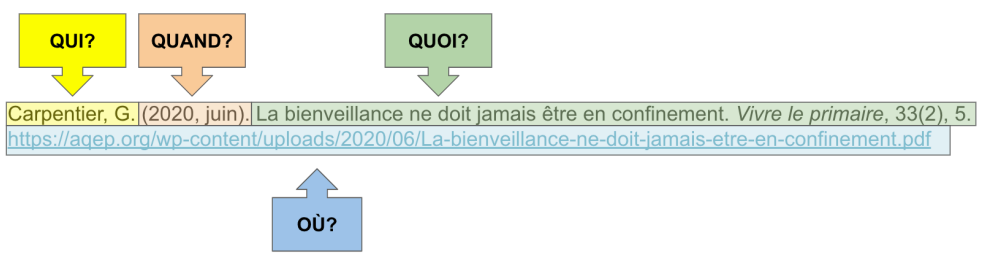

L'exemple ci-dessus est un article dans une revue professionnelle en version électronique sur un site Web.

- Auteur : est l'auteur de l'article. 
- Date : est la date de publication de la revue, qui suit le format (année, jour mois).

- Titre : la première lettre du premier mot est en majuscule.

- Localisation de la source : comprend

1. le titre de la revue professionnelle en italique

1. pour les périodiques en français, mettre une majuscule à la première lettre du premier mot du titre;

2. pour les périodiques en anglais, mettre tous les mots en majuscule sauf les petits mots.

2. Le volume et le numéro entre parenthèses,

3. l'intervalle de pages de l'article et

4. l'adresse URL correspondante en format hyperlien.

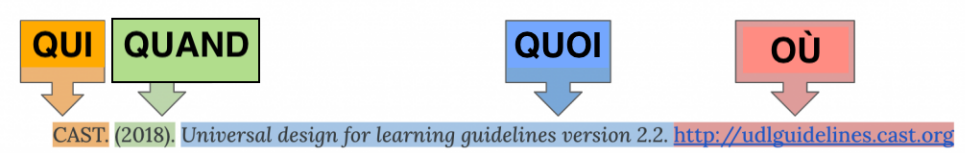

L'exemple ci-dessus est une page Web avec un auteur (nom de l'organisation) identique au titre du site Web.

- Auteur : CAST est le nom complet de l'organisation, pas un acronyme.

- Si l'auteur est un acronyme, épelez le nom en toute lettres.

- Date : la date de publication de la page Web.

- N'utilisez pas la date de droit d'auteur du site Web pour la date.

- Titre : mettre une majuscule à la première lettre du premier mot du titre, en italique, car il s'agit d'un ouvrage autonome.

- Localisation de la source : est l'URL de la page Web. 
- Les références de pages Web incluent généralement le titre du site Web, mais ici l'auteur est le même que le titre, donc seul l'auteur est inclus.

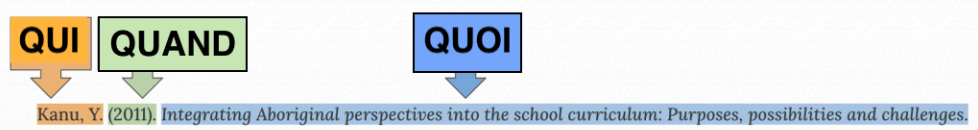

University of Toronto Press.

\section{OÙ}

L'exemple ci-dessus est un livre électronique accessible via une base de données de bibliothèque sans DOI.

- Auteur : est l'auteur du livre.

- Date : la date de droit d'auteur, trouvée sur la page des droits d'auteur (C2011).

- Ceci est la ligne directrice pour les livres imprimés et électroniques. La date de mise ne ligne d'un livre n'est pas utilisée.

- Titre : mettre une majuscule à la première lettre du premier mot du titre, en italique, car il s'agit d'un ouvrage autonome et il comprend un sous-titre séparé par deux-points (:) avec le premier mot en majuscule.

- Localisation de la source : est la maison d'édition du livre. Ce livre n'a pas de DOI et aucune adresse URL n'est incluse, car il se trouve dans une base de données de bibliothèque.

*Si un ouvrage dans une base de données de bibliothèque n'a pas de DOI, votre professeur peut 
préférer avoir une adresse URL. Nous vous conseillons de demander à votre professeur.*

Linfographie ci-dessous donne un aperçu de chacun des 4Q avec quelques exemples (en anglais).

Vous pouvez télécharger l'infographie complète (2 pages) de l'APA 7e édition, présentée tout au long de ce didacticiel, ici : APA 7th Edition Overview Infographic [PDF]. 


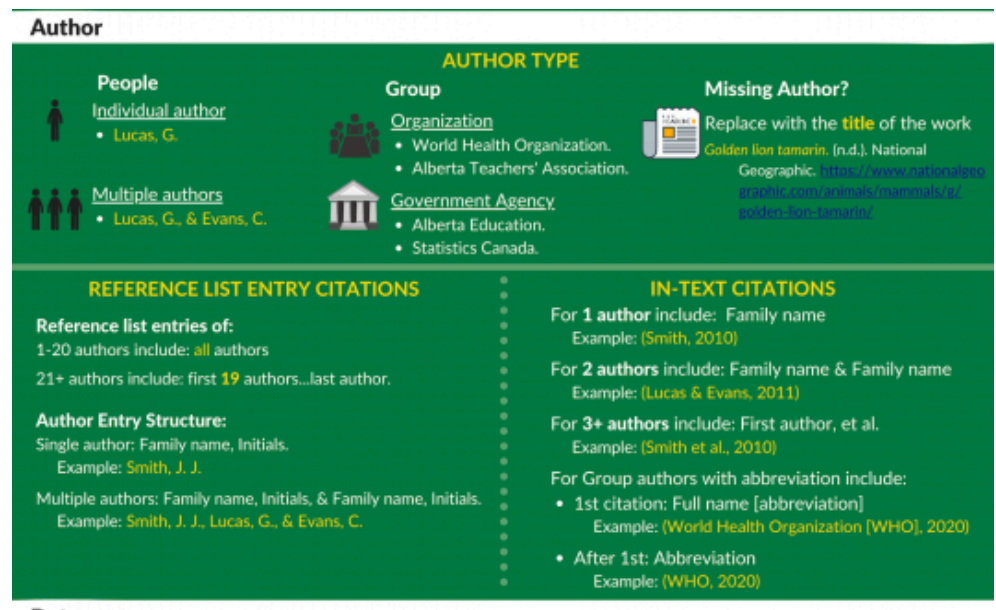

\section{Date}

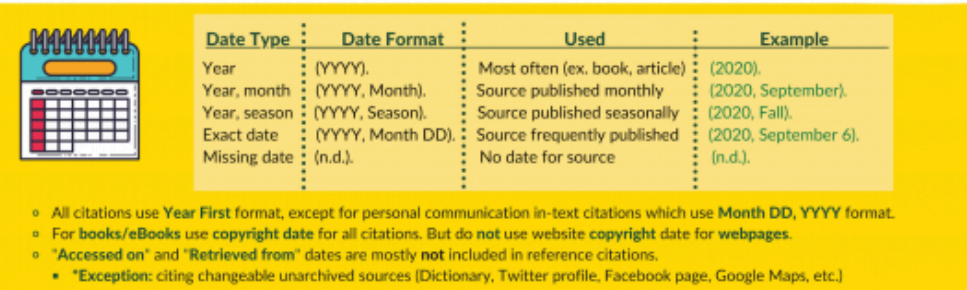

\section{Title}

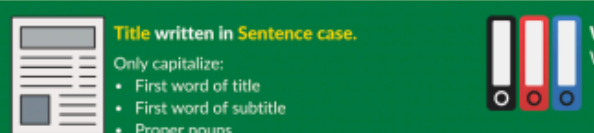

- Proper nouns

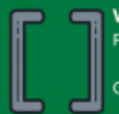

When to use brackets:

For non-textual, non-scholarly works.

- Examples: Multimedia, social media

Citation example - YouTube Video:

Fogarty, M. [Grammar Girl]. [2016, September 30].

How to diogram o sentence (absolute bosics) [Videol. YouTube.

Missing the title?

insert your own [descriptive title]
When to italicize the Work's Title:

When it's a standalone work; most common

- Examples: Books, Reports, Dissertations \&

Theses, Films \& TV Series, Social Media. Webpages, YouTube Video

Citation example - Webpage template: Name. (Date). Webpoge title. Website Title. URL

When to italicize the Work's Source?

When it's a part of another source

- Examples: Article within a Jot

Newspaper or Periodical Article, Edited Book Chapter, Blog Post, TV Episode

Citation example - Joumal Article:

McCauley. 5. M. \& Christiansen. M. H. (2019) Language leaming use: A cross-lingulstic model of child langusze develooment. Psychobatical Review. 12611 1-51.

\section{Source Location}

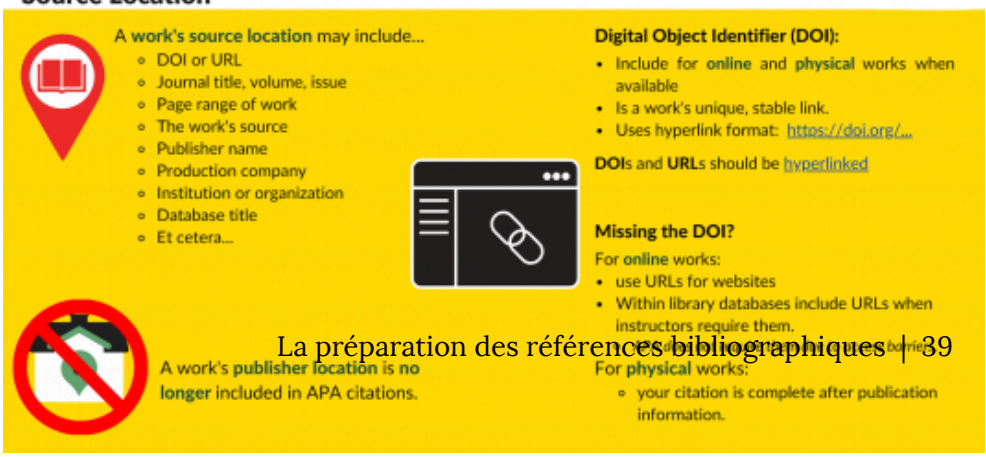




\section{Activité pour la préparation des références}

\section{bibliographiques}

Comme vous l'avez appris, la préparation de références bibliographiques nécessite quelques étapes :

1. Identifier la sorte de source,

2. Identifier les 4 Questions de l'ouvrage (Qui, Quand, Quoi, Où),

3. Rédiger la référence en utilisant les $4 \mathrm{Q}$ et les conseils d'un exemple pertinent (voir Aide-mémoire APA et APA QuickGuide),

4. Enfin, il est également important de relire votre travail.

Lorsque vous créez une référence, faites attention aux $4 \mathrm{Q}$ et au formatage de chaque référence, utilisant correctement la ponctuation et l'emphase de police (italique).

Complétez les activités ci-dessous pour pratiquer la création de références. Vous pouvez cliquer sur n'importe laquelle des images pour les agrandir.

\section{Activité 1}

40 | Activité pour la préparation des références bibliographiques 
Enseigner en milieu francophone minoritaire canadien:

syuthèse des comnaissances sur les défis et leurs

implications pour la formation des enseignants

Mevtine Cavangh

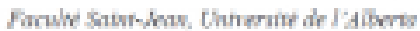

Loment Cammeata

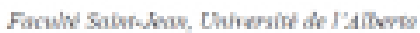

Syhie Blain

Chinerate de Mavcrion

\section{Résunte}

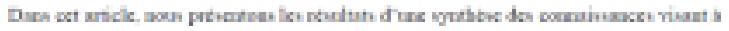

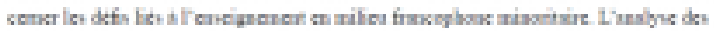

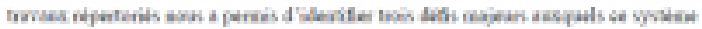

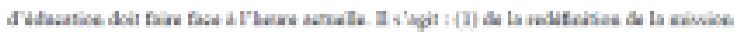

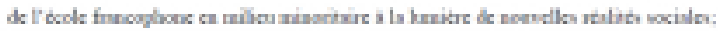

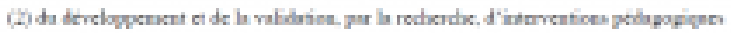

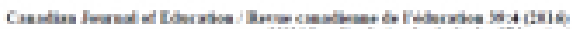

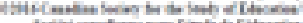

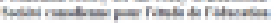

An interactive or media element has been excluded

믓 from this version of the text. You can view it online

here:

https://openeducationalberta.ca/tutoriel-apa/?p=71

\section{Activité 2}




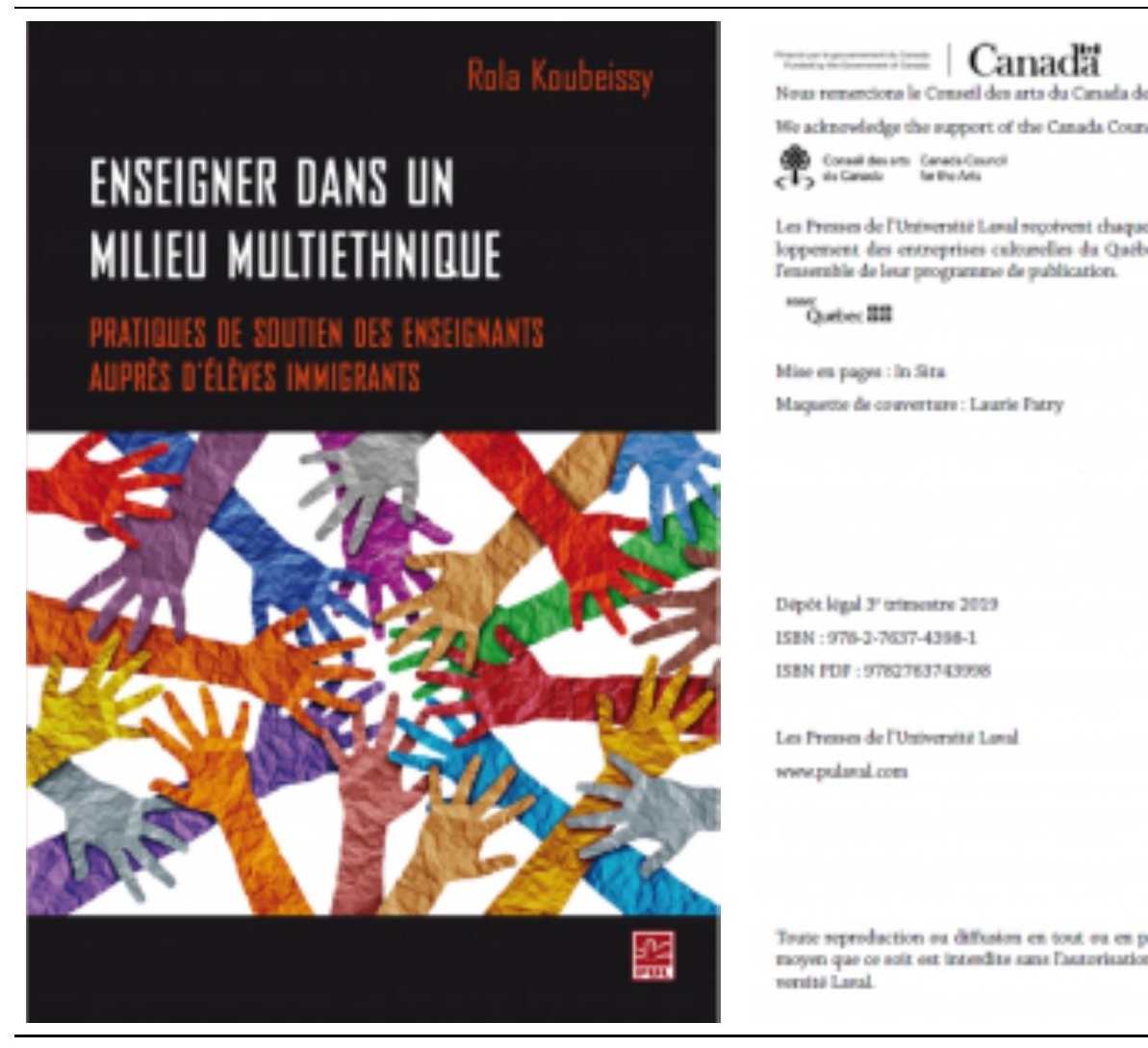

Vous souhaitez créer une référence pour le livre électronique ci-dessus. Faites glisser-déposer chaque élément de la référence dans la case appropriée.

An interactive or media element has been excluded
from this version of the text. You can view it online
https://openeducationalberta.ca/tutoriel-apa/?p=71

42 | Activité pour la préparation des références bibliographiques 


\section{Activité 3}

A rurd leaming momert/ Un moment d apprentiseage en miteu nurd EOTOA'S NOTEBOCK I NOTE OE LA AEDACTNCE

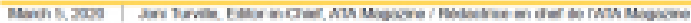

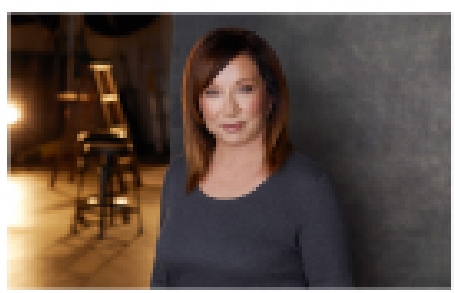

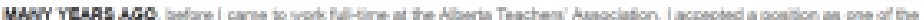

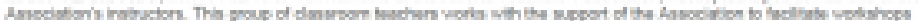

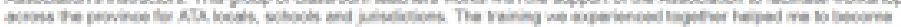

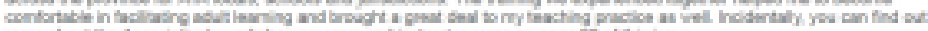

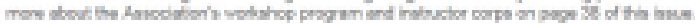

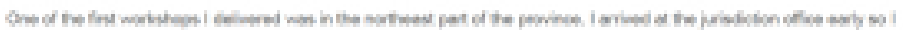

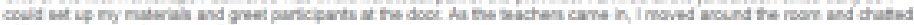
veth as mary peode in poanble.

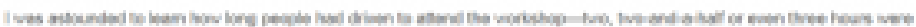

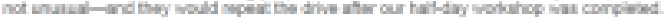

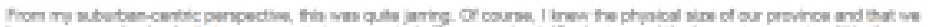

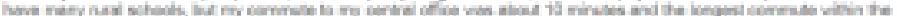

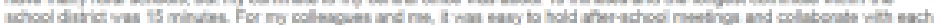

\section{Activité 4}


Serge Manhand. 0je Sarave. latelie Gauned

Preface de Antorio Danavio

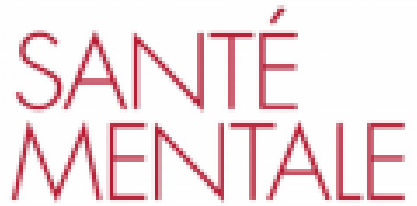

\section{ET DOULEUR}

Compscantes somatieues et psychiatriąes

de la douleur en sante mentale

\section{Santé mentale et doul}

Composantes somatiques et psychiatriques de la douleur en santé me

Q Springer 


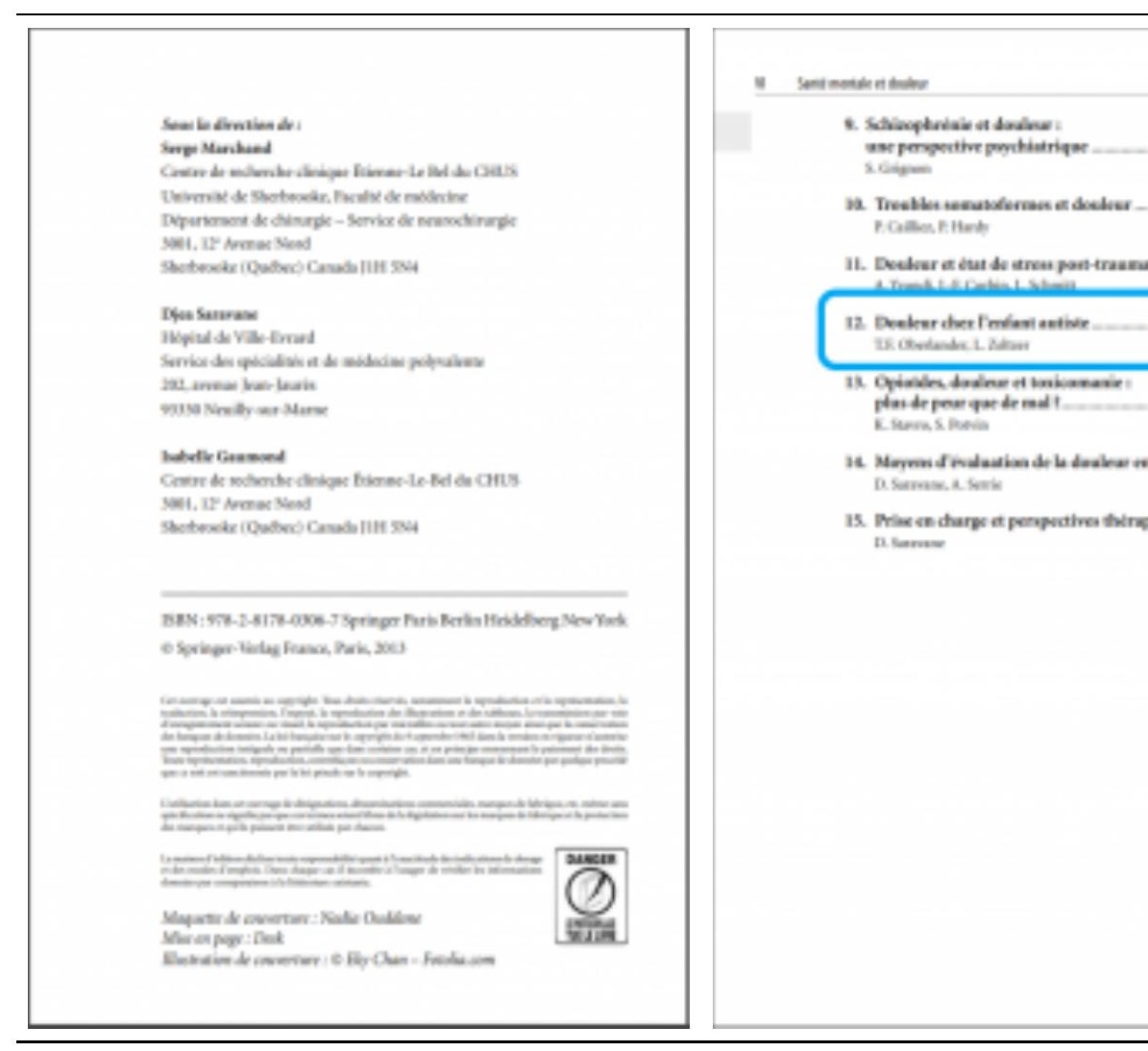

An interactive or media element has been excluded
from this version of the text. You can view it online here:

https://openeducationalberta.ca/tutoriel-apa/?p=71 

PART IV

CITATIONS DANS LE TEXTE

Citations dans le texte | 47 



\section{Qu'est-ce qu'une citation ou un renvoi dans le texte?}

Résultat d'apprentissage

A la suite de ce chapitre vous pourrez :

- $\quad$ Créer et formater une citation dans le texte dans le style APA.

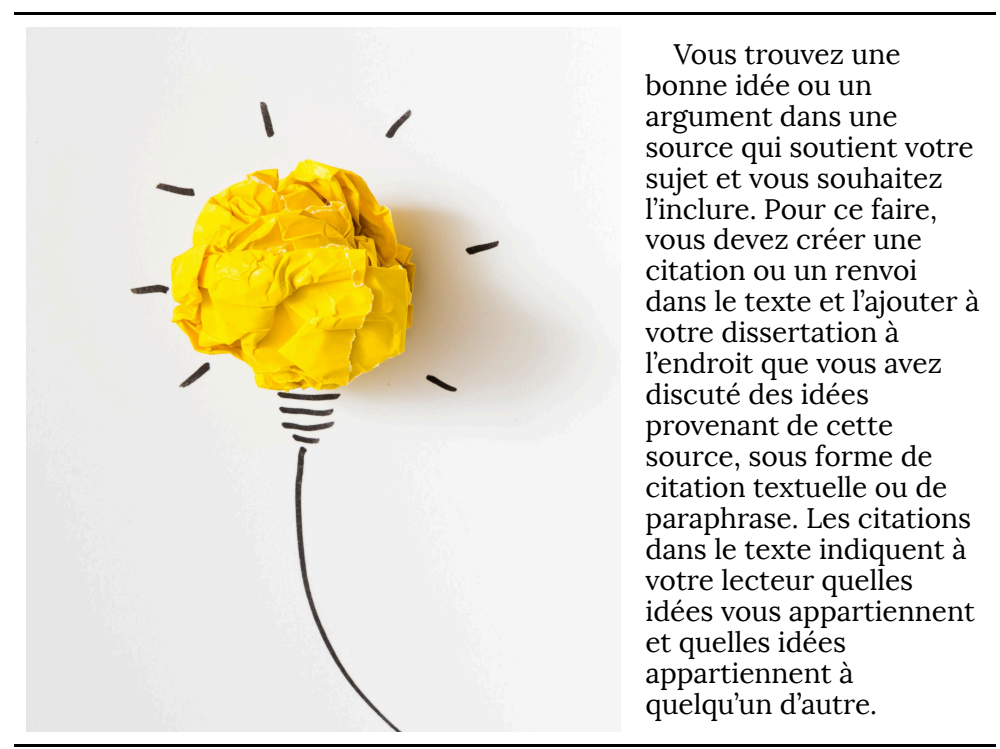

Il existe deux façons différentes d'inclure des citations ou des 
renvois dans le texte : en tant que citation entre parenthèses ou citation narrative.

Lexemple suivant montre comment inclure ces deux types de renvoi dans un article. Cliquez sur le symbole $\mathbf{D}$ pour en savoir plus.

An interactive or media element has been excluded

무스 from this version of the text. You can view it online

here:

https://openeducationalberta.ca/tutoriel-apa/?p=78

L'image ci-dessous donne un aperçu des deux sortes de renvoi.

\begin{tabular}{|c|c|}
\hline \multicolumn{2}{|c|}{$\sqrt{\square}$ Deux manières d'insérer un renvoi ou une citation: } \\
\hline Citation entre parenthèses & Citation narrative \\
\hline $\begin{array}{l}\text { 1- Nom de farmille de lauteur et llannée } \\
\text { de publication sont insérés à la fin d'une } \\
\text { phrase entre parenthèses. }\end{array}$ & $\begin{array}{l}\text { 1- Le nom de famille de lauteur fait partie de la phrase } \\
\text { suivi de lamnée de publication de l'ouvrage cité entre } \\
\text { parenthèses. }\end{array}$ \\
\hline $\begin{array}{l}\text { Exemple, ... la construction du sens } \\
\text { (Dagenais et Toohey, 2014). }\end{array}$ & $\begin{array}{l}\text { Exemple, Lebrun et ses collaborateurs (2012) estiment } \\
\text { que ... }\end{array}$ \\
\hline & $\begin{array}{l}\text { 2- L'année de publication peut aussi faire partie de la } \\
\text { phrase }\end{array}$ \\
\hline & $\begin{array}{l}\text { Exemple, En 2012, Lebrun et ses collaborateurs ont } \\
\text { estimé que... }\end{array}$ \\
\hline
\end{tabular}

Comme vous pouvez le voir, une citation dans le texte est formatée à l'aide de trois éléments clés :

1. Parenthèses,

2. Le nom de famille ou le nom du groupe de l'auteur, et

3. L'année.

Les informations de citations sont tirées directement de la référence bibliographique correspondante. Ainsi, il est plus facile

50 | Qu'est-ce qu'une citation ou un renvoi dans le texte? 
de créer d'abord la référence, ensuite la citation dans le texte qui l'accompagne.

Lebrun, M., Lacelle, N. et Boutin, J.-F. (dir). (2012). La littératie médiatique multimodale. De nouvelles approches en lecture-écriture à l'école et hors de l'école. Presses de l’Université du Québec.

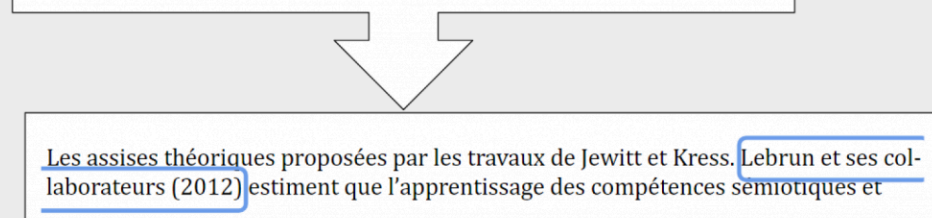

Jusqu'à présent, nous nous sommes concentrés sur des exemples paraphrasées. Maintenant, nous allons examiner des exemples de citations textuelles pour les citations dans le texte.

Lorsque vous faites une citation textuelle au lieu d'une paraphrase, vous devez également inclure l'emplacement de la citation dans l'œuvre. De plus, lorsque vous paraphrasez des passages spécifiques dans des œuvres plus longues, vous incluez l'emplacement. Les informations de localisation sont ajoutées à votre référence dans le texte directement après la date. Par exemple, une citation entre parenthèses ressemblerait à : (Smith, 2010, p. 3-4).

Notez que les professeurs préfèrent souvent avoir les informations de localisation pour toutes les citations dans le texte, alors vérifiez auprès de votre professeur.

Pour les exemples de citations textuelles suivants, cliquez sur le 
symbole $\mathbf{1}$ pour savoir comment ajouter des citations dans le texte pour les extraits courts et les extraits en blocs de texte.

\section{Exemples d'extrait court}

Citation narrative
圆
An interactive or media element has been excluded from this version of the text. You can view it online here:

https://openeducationalberta.ca/tutoriel-apa/?p=78

Citation entre parenthèses

An interactive or media element has been excluded
here:
https://openeducationalberta.ca/tutoriel-apa/?p=78

\section{Exemples d'extrait en bloc de texte}

Citation narrative

An interactive or media element has been excluded
here:
https://openeducationalberta.ca/tutoriel-apa/?p=78

Citation entre parenthèses

52 Qu'est-ce qu'une citation ou un renvoi dans le texte? 


\section{An interactive or media element has been excluded}

from this version of the text. You can view it online

here:

https://openeducationalberta.ca/tutoriel-apa/?p=78

L'image ci-dessous (avec texte en anglais) donne des exemples de localisation et des abréviations appropriées. Voir le tableau 1 de l'Adaptation française des normes bibliographiques de l'APA pour savoir quelles sont les différences entre les normes bibliographiques anglaises et françaises.

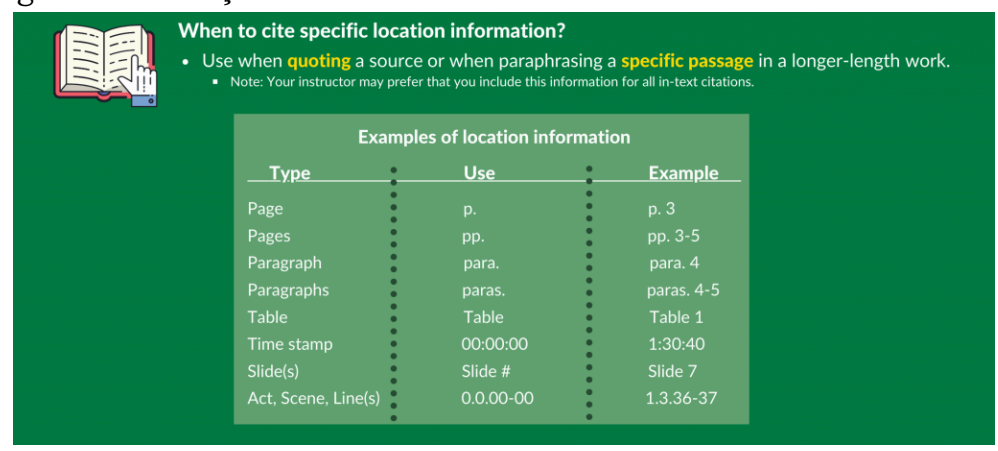

Notez que les professeurs préfèrent souvent avoir les informations de localisation pour toutes les citations dans le texte, alors vérifiez auprès de votre professeur.

Maintenant que nous avons vu les principes de base des citations dans le texte, passez à la section suivante pour effectuer quelques activités pratiques. 
Attribution d'image:

"Illuminated crumpled yellow paper light bulb idea on white background Free Photo" by Freepik is licensed under Freepik Licence, attribution required.

54 | Qu'est-ce qu'une citation ou un renvoi dans le texte? 


\section{Activité pour les citations dans le texte}

Dans cette section, complétez les trois activités suivantes pour pratiquer la création des citations entre parenthèses et narratives dans le texte en tapant la réponse dans le champ texte.

国 An interactive or media element has been excluded here:

https://openeducationalberta.ca/tutoriel-apa/?p=80 


\section{Solutions à l'activité pour les citation dans le texte}

\section{Question 1:}

Depuis l'adoption de l'article 23 de la Charte canadienne des droits et libertés, en 1982, les parents membres de la minorité francophone de la province où ils résident, qui répondent à des critères spécifiques associés à cet article, ont le droit de faire instruire leurs enfants en français (Faucher, 1999).

\section{Question 2:}

Plus tard, Blain et Lowe (2009) se sont intéressées à cette même problématique du développement conjoint des compétences langagières et de l'identité francophone en sondant des enseignants et des élèves néo-Brunswickois pour savoir quelles pratiques pédagogiques en classe de français favorisent l'apprentissage de l'oral tout en stimulant des sentiments d'appartenance.

\section{Question 3:}

De façon générale, nous avons été surpris de constater que la plus grande lacune se situe dans le domaine de la formation initiale des futurs enseignants qui pourtant s'avère être le «vecteur de transformation des cultures éducatives et professionnelles » (Forget et Sabatier, 2014, p. 125). 
PART V

\section{COMMENT TOUT \\ FONCTIONNE ENSEMBLE}





\section{Comment tout fonctionne ensemble}

Après avoir terminé ce chapitre, vous serez en mesure de

- Créer et formater une liste de références bibliographiques et des citations dans le texte dans le style APA.

Comme nous l'avons vu, les références bibliographiques et les citations dans le texte doivent correspondre l'une à l'autre. Elles travaillent ensemble pour indiquer clairement quelles sources soutiennent votre devoir. 
understanding of the local context Fulcher, Davidson, and Kemp (2011) hote the following: Measurement-driven scales suffer from descriptio al inadequacy. They are not sensitive to the communicative context or the interactior al complexities of language use. The level of abstraction is too great, creating a gulf $b$ tween the score and its meaning. Only with a richer description of contextually bas d performance, can we strengthen the meaning of the score, and hence the validity of score-based inferences. (pp. 8-9)

References

Fulcher, G., Davidson, F., \& Kemp, J. (2011). Effective rating scale development for speaking tests: Performance decision trees. Language Testing, 28(1), 5-29.

https://doi.org/10.1177/0265532209359514

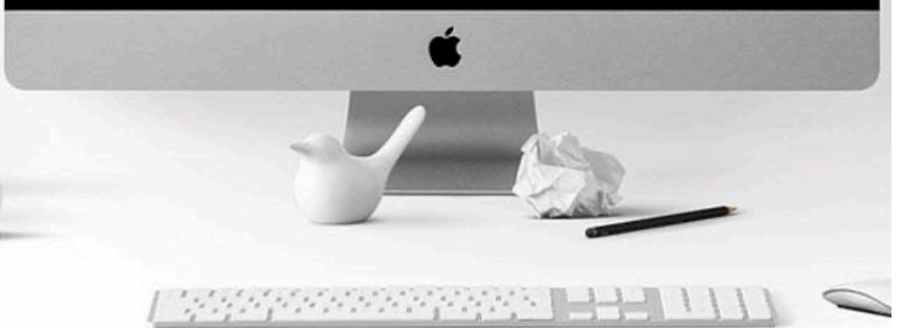




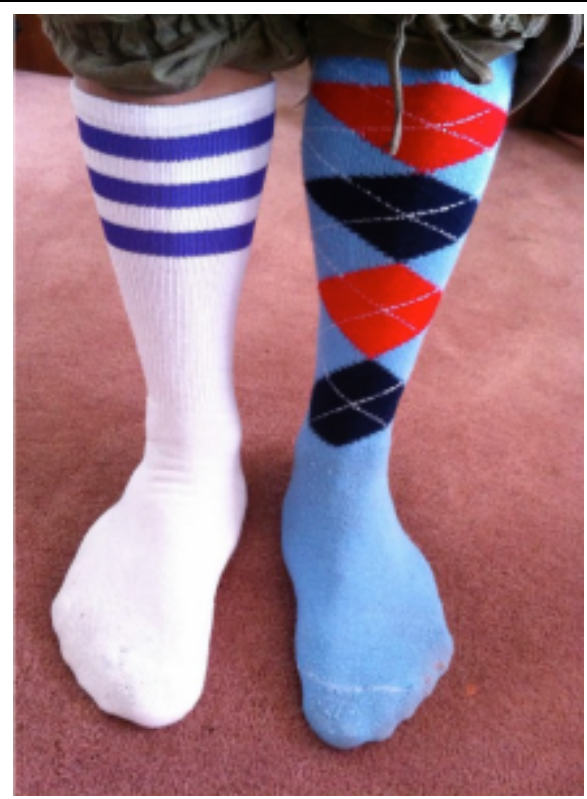

Vous ne voudriez pas porter des chaussettes dépareillées, tout comme vous ne voulez pas de citations mal assorties!

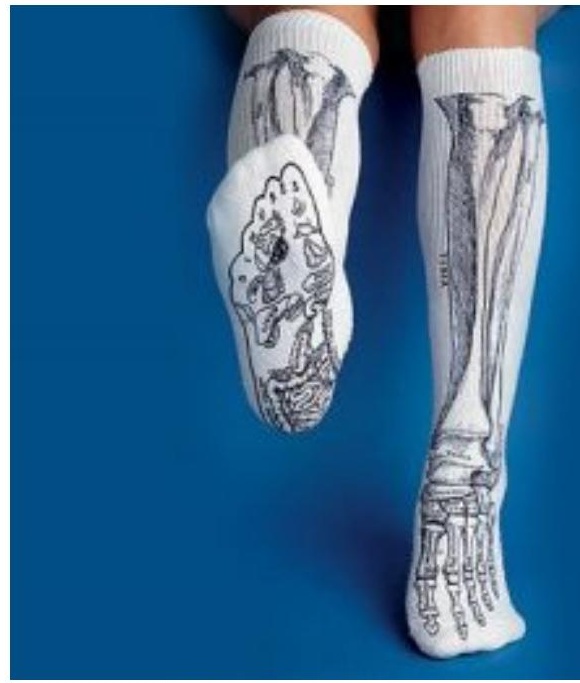

Vous voulez que vos citations (et chaussett et dans la liste de références bibliogr correspondent l'une à l'autre

*Une exception est la communication personnelle, qui est citée comme citation dans le texte, mais n'est pas incluse dans la liste des références bibliographiques.*

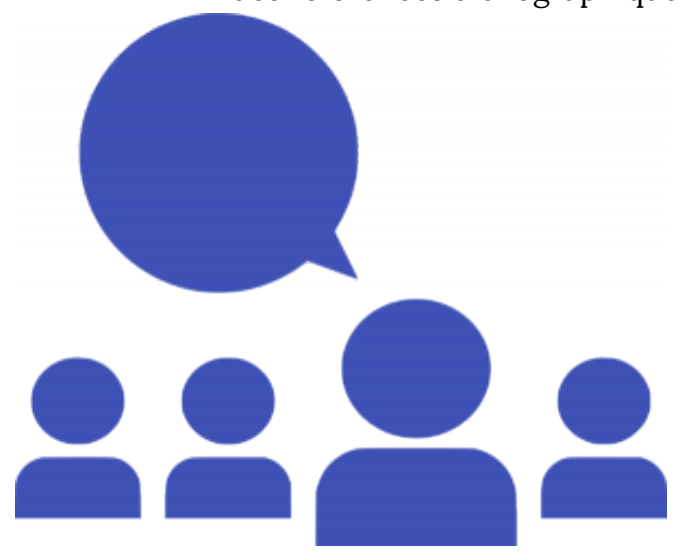

Comment tout fonctionne ensemble $\mid 61$ 
Pourquoi? Votre lecteur ne peut pas accéder à vos expériences personnelles.

Dans l'activité suivante, vous allez pratiquer à faire correspondre les citations dans le texte avec leur références bibliographiques.

Attribution d'images:

"[In-Text Citation Example in Apple MAC Desktop Computer]" from NorQuest College Library's Intro to APA tutorials is licensed under CC BY-NC-SA 4.0 International / Added updated reference and in-text citation images.

"Mismatched Socks Solidarity Day" by Rik Panganiban is licensed under CC BYNC-SA 4.0 International.

"X-ray socks" by unknown author is licensed under $\mathrm{CC}$ BY-NC-SA 4.0 International.

62 | Comment tout fonctionne ensemble 


\section{Activité de couplage des références bibliographiques et des citations dans le texte}

Pour les quatre questions suivantes, associez la référence bibliographique à la citation dans le texte correspondante en choisissant la réponse appropriée.

Cormier, M., Pruneau, D., et Rivard, L. (2010). Améliorer les apprentissages en sciences en milieu francophone minoritaire : résultats de l'expérimentation d'un modèle pédagogique. Revue des sciences de l'éducation, 36(2), 343-363.

https://doi-org/10.7202/044481ar

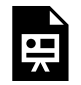

An interactive or media element has been excluded here: from this version of the text. You can view it online

https://openeducationalberta.ca/tutoriel-apa/?p=93

Fédération des conseils scolaires francophones de l'Alberta. (s.d). Education francophone. https://fcsfa.ca/education-francophone/ 
An interactive or media element has been excluded

긋 from this version of the text. You can view it online here:

https://openeducationalberta.ca/tutoriel-apa/?p=93

Tardif, C. (2020, été). Chronique : La voix du primaire. Musique et Pédagogie, 34(3), 20-21. http://www.fameq.org/sites/default/files/revues-pdfs/fameq 15306 revue vol 34 no3 - v2.pdf

An interactive or media element has been excluded

믓 from this version of the text. You can view it online here:

https://openeducationalberta.ca/tutoriel-apa/?p=93

Champy, P., et Etévé, C. (2005). Dictionnaire encyclopédique de l'éducation et de la formation (3e éd.). Retz.

固 An interactive or media element has been excluded here:

https://openeducationalberta.ca/tutoriel-apa/?p=93

64 | Activité de couplage des références bibliographiques et des citations dans le texte 


\section{APA et aide à la rédaction}

Résultat d'apprentissage

Après avoir terminé ce chapitre, vous pourrez :

- $\quad$ Accéder aux ressources disponibles pour vous aider avec la citation dans le style APA.

L'Université de l'Alberta a plusieurs ressources à votre disposition pour vous aider avec la citation dans le style APA, les services et ressources de la bibliothèque, et l'aide à la rédaction.

Citer dans le style APA

Le guide de Citations (français) de la bibliothèque contient des guides et outils pour vous aider avec le style APA, notamment l'aide mémoire APA, l'Adaptation française des normes bibliographiques de l'APA, Purdue OWL (en anglais), etc.

Le site Web officiel APA Style and Grammar Guidelines n'existe pas en français, mais présente de nombreuses recommandations et ressources utiles. Le site Web officiel de l'APA présente aussi des informations sur la manière de citer une source citée 
par une autre que l'on a consulté (voir aussi p. 7 de l'Adaptation française des normes bibliographiques de l'APA).

\section{Tutoriels de base}

La bibliothèque a des tutoriels (en français) pour vous aider lors de vos recherches. Chaque tutoriel est une courte vidéo sur un sujet particulier pour vous aider à développer une stratégie de recherche, à découvrir des différentes sortes de sources, et à développer votre parcours de recherche.

\section{Guide de recherche en Education}

Besoin d'aide pour trouver des ressources ou identifier quelles bases de données à utiliser pour vos devoirs? Consultez le Guide de recherche en Education.

\section{Posez-nous une question}

Si vous avez des questions, le personnel de la bibliothèque est là pour vous aider! Cliquez Question sur le site Web de la bibliothèque pour clavarder avec le 
personnel de la bibliothèque, envoyer un courriel ou prendre rendez-vous avec un bibliothécaire.

\section{Aide à la rédaction et à la recherche}

Pour obtenir de l'aide à la rédaction et à la recherche à l'Université de l'Alberta, visitez la page Web de La Centrale (Campus Saint-Jean) et la page Web des services aux étudiants (en anglais). 


\section{Conclusion}

Merci d'avoir suivi le Tutoriel d'introduction au style de citation APA!

N'hésitez pas à le re-consulter aussi souvent que vous en aurez besoin.

Rappel : Le personnel de la Bibliothèque de l'Université de l'Alberta se fera un plaisir de répondre à vos questions sur les normes de citation ou sur nos services et ressources. 


\section{Références bibliographiques et attribution}

\section{Références}

Barnes, J. (2018). Applying cross-curricular approaches creatively. Routledge. https://doi.org/10.4324/9781315513614

CAST. (2018). Universal design for learning guidelines version 2.2. http://udlguidelines.cast.org/

Centre for the Study of Historical Consciousness. (n.d.). Historical thinking concepts. Historical Thinking Project. http://historicalthinking.ca/historical-thinking-concepts

Creekpaum, S. (2019). Child development through play. In M. Charles \& J. Bellinson (Eds.), The importance of play in early childhood education: Psychoanalytic, attachment, and developmental perspectives (pp. 11-18). Routledge. https://doi.org/ $\underline{10.4324 / 9781315180090}$

Ewasiuk, K. (2012, Summer). What do I do all day? ATA Magazine, 92(4), 14.

Godin, K. M., Patte, K. A., \& Leatherdale, S. T. (2018, February). Examining predictors of breakfast skipping and breakfast program use among secondary school students in the COMPASS study. Journal of School Health, 88(2), 150-158. https://doi.org/ 10.1111/josh.12590

Kanu, Y. (2011). Integrating Aboriginal perspectives into the school curriculum: Purposes, possibilities and challenges. University of Toronto Press.

Kirkpatrick, L., Brown, H. M., Searle, M., Smyth, R. E., Ready, E. A., \& Kennedy, K. (2018). Impact of a one-to-one iPad initiative on Grade 7 students' achievement in language arts, mathematics, and learning skills. Computers in the Schools, 35(3), 171-185. https://doi.org/10.1080/07380569.2018.1491771

McRae, P. (2020, Winter). Ambiguous but gaining momentum. ATA 
Magazine, 100(2), 30-32. https://www.teachers.ab.ca/ SiteCollectionDocuments/ATA/ATA Magazine/Vol 100/ Vol100-No2-Winter 2020.pdf

Purdue Online Writing Lab. (n.d.). APA sample paper. https://owl.purdue.edu/owl/research and citation/ apa style/apa formatting and style guide/ apa sample paper.html

Scribbr. (2019, November 26). APA manual 7th edition: 17 most notable changes | Scribbr [Video]. YouTube. https://www.youtube.com/ watch?v=zeSIXD6y3WQ

Sensoy, Ö., \& DiAngelo, R. (2017). Is everyone really equal?: An introduction to key concepts in social justice education (2nd ed.). Teachers College Press.

Stone, A. (2020, January/February). The end of discipline in the classroom. Teach, 26-29. https://issuu.com/teachmag/docs/ teach janfeb2020

Media Attributions

"book-collage tcm11-262933 w1024_n" by American

Psychological Association.

"Silhouette Head Bookshelf Knowledge" by Gerd Altmann is licensed under Pixabay Licence, no attribution required.

"[What exactly is citing summary]" from NorQuest College Library's Intro to APA tutorials is licensed under CC BY-NC-SA 4.0 International.

"[Publication Manual of the APA, 7th Edition Book Cover]" by American Psychological Association.

"[Smith and Gould In-text Citation Example]" from NorQuest College Library's Intro to APA tutorials is licensed under CC BY-NCSA 4.0 International / Border added and refocused.

"write-writer-type-machine-creative-idea" by mohamed hassan is licensed under CC0 1.0 International.

"[Calendar Months]" by unknown author is licensed under $\underline{\mathrm{CC} 01.0}$ International. 
"stack-of-food-books-2" by Cannelle is licensed under CC BY 4.0 International.

“512px-Internet2" by Fabio Lanari is licensed under CC BY-SA 4.0 International.

"Pancake recipe with ingredients Free Vector" by pikisuperstar is licensed under CC BY 4.0 International.

"[Journal article publication and summary information]" from NorQuest College Library's Intro to APA tutorials is licensed under CC BY-NC-SA 4.0 International.

"Illuminated crumpled yellow paper light bulb idea on white background Free Photo" by Freepik is licensed under Freepik Licence, attribution required.

"[In-Text Citation Example in Apple MAC Desktop Computer]" from NorQuest College Library's Intro to APA tutorials is licensed under $\mathrm{CC}$ BY-NC-SA 4.0 International / Added updated reference and in-text citation images.

"Mismatched Socks Solidarity Day" by Rik Panganiban is licensed under CC BY-NC-SA 4.0 International.

"X-ray socks" by unknown author is licensed under $\underline{\mathrm{CC} B Y-\mathrm{NC}-\mathrm{SA}}$ 4.0 International.

"Opinion" by unknown author is licensed under $\mathrm{CCO} \quad 1.0$ International.

All other images in this tutorial are created by the University of Alberta Library. The use of these images should be credited to the University of Alberta Library using the Creative Commons Licence CC BY-NC-SA 4.0 International. 